\title{
Bernard, Sylvain, Michel Farge (edd.): Les mutations contemporaines du droit de la famille
}

\author{
Grenoble, Presses Universitaires de Grenoble, coll. « Droit et action \\ publique», 2019, 212 p, $€ 27$ (Paperback), ISBN 978-2-7061-4410-3
}

\section{Paulina Mazurkiewicz ${ }^{1}$ iD}

Accepted: 26 January 2021 / Published online: 25 February 2021

(c) The Author(s) 2021, corrected publication 2021

L'ouvrage collectif Les mutations contemporaines du droit de la famille dirigé par Sylvain Bernard et Michel Farge s'assigne pour tâche de présenter une réflexion d'ensemble sur les transformations du droit de la famille ces dernières années. Les auteurs de treize contributions revisitent le sujet des mutations à travers les notions telles que la filiation (et sa libéralisation avec les procédés de la PMA et la GPA) et le couple dans la perspective du divorce. L'ensemble est divisé selon deux fonctions du droit de la famille: symbolique ou anthropologique (partie 1) et opératoire ou judiciaire (partie 2).

La première partie rassemble les textes portant sur la question du sexe des individus et la filiation. Tout d'abord, Loïc Bret étudie la question du sexe et du genre dans le droit français et européen. Selon lui, la définition juridique du terme sexe, à l'opposition de l'information médicale contenue dans l'acte de naissance, ne « présenterait aucune utilité» [p. 11]. Chacun se définirait à partir de son propre ressenti, d'où l'apparition du concept 'identité de genre' admis par l'Assemblée parlementaire du Conseil de l'Europe. Pourtant, malgré des pressions de plusieurs milieux, la démédicalisation et la déjudiciarisation de la procédure de changement de sexe à l'état civil ne sont pas encore décidées par la Cour européenne. De plus, le droit français refuse continuellement la reconnaissance de l'intersexuation au sein de l'état civil ce qui pousse certains auteurs à militer pour l'effacement de la mention de sexe à l'état civil.

Jennifer Naselli, pour sa part, examine l'état du droit positif vingt-cinq ans après l'entrée en vigueur de la première loi de bioéthique concernant l'assistance médicale à la procréation ou la procréation médicalement assistée (AMP ou PMA) et de la gestation pour autrui (GPA). Le premier procédé, comme moyen de créer artificiellement ce qui ne peut naturellement être conçu, se résume en techniques médicales

Paulina Mazurkiewicz

paulina.mazurkiewicz@kul.pl

1 Université Catholique de Lublin Jean-Paul II, Lublin, Poland 
« permettant la conception in vitro, la conservation des gamètes, des tissus germinaux et des embryons, le transfert des embryons et l'insémination artificielle» [p. 23]. La gestation pour autrui est interdite en France selon le Code civil (art. 16-7) vu le risque de réification de l'enfant à naître et de la femme en tant que mère porteuse. L'AMP est accessible sous certaines conditions comme: le caractère hétérosexuel du couple consentant, la garantie à l'enfant d'un accueil dans une famille afin d'assurer des conditions optimales pour son développement personnel. En outre, chacun des futurs parents doit être en âge de procréer et être vivant. Le consentement à l'AMP exprimé individuellement doit être écrit, libre et éclairé (C. civ., art. 311-20). Quoique le motif fondamental de cette technique soit médical (compenser l'infertilité naturelle d'un couple hétérosexuel), l'Auteure observe des revendications à d'autres fins: l'assistance médicale à la procréation serait un remède à « l'infertilité sociale». Elle fait remarquer également le problème de filiation lié à l'AMP avec un tiers donneur et le tourisme procréatif découlant de l'interdiction française de la maternité de substitution. Les discussions autour des procédés en question, influencées également par la jurisprudence européenne, font établir de nouvelles institutions telles que la Cour de réexamen des décisions civiles françaises en matière d'état des personnes (créée par la loi 2016-1647).

Jehanne Sosson et Hélène Malmanche présentent la problématique des PMA et GPA dans la perspective du droit belge largement ouvert à ces procédés (possibilité donnée aux célibataires, aux couples mariés ou non, hétéro et homosexuels, aussi étrangers, notamment français) grâce à la loi du 6 juillet 2007. Elle garantit également la gratuité et l'anonymat du don de gamètes. Par contre, elle ne réglemente pas l'établissement des liens juridiques de filiation suite aux processus de procréation médicalement assistée (sauf les art. 27 et 56 de la loi qui traitent de la filiation et se limitent à déclarer que « les règles de la filiation telles qu'établies par le Code civil jouent en faveur du ou des auteurs du projet parental»). Les Auteures admettent que ce modèle belge, ouvrant l'accès large à la PMA et à la GPA permettant à assurer la filiation des parents d'intention et leurs enfants nés par gestation pour autrui, diverge diamétralement du modèle français où le législateur circonscrit l'accès à la PMA et défend la GPA ou encore du modèle américain permettant l'intervention d'argent dans ces procédés.

Géraldine Vial, pour sa part, aborde la procédure menée à la Cour de réexamen des décisions civiles en matière de gestation pour autrui « marquant une nouvelle étape dans la saga des enfants nés à l'étranger par une gestation pour autrui» [p. 61]. Selon les articles L. 452-1 et suivants du Code de l'organisation judiciaire, la saisine de la Cour de réexamen doit répondre à certaines conditions: elle doit être réalisée par « une personne ayant été partie à l'insistance et disposant d'un intérêt» [p. 63]. La décision doit être définitive, irrévocable et prononcée en violation de la Convention de sauvegarde des Droits de l'Homme et des Libertés fondamentales (ESDH) et condamnée par la Cour européenne des droits de l'homme (EDH). Il est encore indispensable que cette violation de la Convention ESDH entraîne « par sa nature et sa gravité» pour le requérant « des conséquences dommageables auxquelles la satisfaction équitable ne pourrait mettre un terme» (art. L. 452-1 et s., Code de l'organisation judiciaire). Le réexamen doit, enfin, être demandé dans un délai d'un an après la décision de la Cour EDH. La procédure de réexamen comporte deux 
étapes qui se suivent: la recevabilité de la demande et son bien-fondé. Quant au domaine de la compétence de la Cour, il est doublement limité: par le temps (délai d'un an après la décision de la Cour EDH) et par son objet (« en matière d'état des personnes», art. L. 452-1 et s., Code de l'organisation judiciaire). L'Auteure conclut ses propos en énonçant que le réexamen des décisions civiles « autorise une meilleure effectivité des décisions de la Cour européenne dans le droit français, mais, de l'autre côté, il paraît « désormais placer le choix de notre société directement sous l'égide de la Cour européenne» [p. 68].

La seconde partie de l'ouvrage se concentre autour de la déjudiciarisation du droit de la famille: divorce conventionnel sans juge et d'autres reformes concernant certaines conséquences du divorce. Nicolas Frémeaux examine la question du déclin du mariage depuis le début des années 1970 causé, entre autres, par le phénomène de la cohabitation (changements de normes sociales et religieuses, rallongement des études en sont des causes principales). Depuis 1999 existe en France une alternative au mariage, le pacte civil de solidarité qui donne plus de droits (par exemple, relatifs à la fiscalité) que l'union libre. Quant au patrimoine, les couples mariés choisissent entre la communauté des biens (le régime par défaut) et la séparation des biens (un régime conventionnel). L'Auteur remarque une hausse récente du recours au contrat de mariage avec la séparation des biens comme option souvent choisie qui donne la possibilité de s'assurer contre le risque de divorce. En ce qui concerne les évolutions des pratiques matrimoniales, l'Auteur affirme que les couples mariés ont des patrimoines statistiquement plus élevés que les couples non mariés. De plus, les couples ayant opté pour la séparation des biens sont considérablement plus riches que les couples non mariés et qu'on y observe la proportion plus élevée des travailleurs indépendants. Il est également à noter que les personnes divorcées constituent $18,1 \%$ des 465450 personnes qui se sont mariées en 2016 et que, plusieurs études le confirment, la diminution de niveau de vie suite au divorce touche davantage les femmes que les hommes.

Ingrid Maria aborde le problème du divorce sans juge en rappelant d'abord l'histoire du divorce. Les Romains le pratiquaient sous la forme du consentement mutuel et de la répudiation. La conception chrétienne a apporté la conception de l'indissolubilité du mariage, mais n'a jamais parvint à abolir le divorce à Rome. Le protestantisme et la philosophie des Lumières introduisirent le divorce dans la législation française grâce à la conception du mariage comme contrat possible à rompre conformément au principe de liberté individuelle. Il est notable que la loi de 1792 ait permis de divorcer par consentement mutuel devant non pas un juge, mais devant l'officier d'état civil. Le Code Napoléon a restreint par judiciarisation, pendant plus de deux siècles, l'accès au divorce. La discussion, après des pressions régulières, a été de nouveau entamée à l'occasion de l'adoption de la réforme du 26 mai 2004 dont le projet initial prévoyait le privilège des divorces amiables contre le divorce par consentement mutuel au contrôle du juge. Finalement, « la fameuse loi dite $\mathrm{J} 21$ du 18 novembre 2016 censée 'moderniser' la justice du XXI ${ }^{\mathrm{e}}$ siècle, optait pour un divorce sans juge (divorce par consentement mutuel) dans son article 50» [p. 89]. Son objectif principal consistait en réduction des délais de traitement des divorces par consentement mutuel. Suite au rapport Agostini et Molfessis relatif à l'amélioration et à la simplification de la procédure civile, la procédure de divorce 
est soumise à la procédure du droit commun, la loi de programmation pour la Justice 2018-2022 supprime la phase de conciliation. L'Auteure conclut ses remarques en constatant que « le chantier relatif au divorce n'est donc pas près de s'achever» [p. 98]. Dans une autre étude, elle examine les pouvoirs du juge de l'exécution (JEX) sur le titre contractuel en soulignant que si la compétence du JEX pour statuer sur la régularité des actes notaires est indéniable, marquant ainsi la différence avec la remise en cause d'une décision de justice, elle n'est peut-être pas tant à redouter que cela. Si variés puissent être les motifs de remise en cause de l'acte notaire invoqués, l'étude menée démontre que leurs chances de succès sont moindres. Par ailleurs, le JEX n'a pas tous les pouvoirs lorsqu'il statue sur la question de la régularité du titre notaire fondant les poursuites.

Mikaël Gros s'intéresse aux causes, issues du droit commun des contrats, qui entraîneront la nullité de la convention du divorce déjudiciarisé (certains à l'initiative des époux, d'autres à l'initiative de leurs créanciers) et aux effets qu'aura cette nullité sur la convention (son étendue) et sur le principe même du divorce (l'adjonction d'une phrase à l'art. 229-1 prévoyant que « le principe du divorce est alors acquis de façon irrévocable» permettrait de lever les incertitudes sur le caractère définitif du divorce).

Frédéric Hébert et Jean-Luc Charras établissent un bilan, de la perspective du notaire, sur la pratique du divorce sans juge. Il s'étend autour des axes suivants: l'état liquidatif, le dépôt de la convention, la force exécutoire, la publicité foncière. Les Auteurs répondent ainsi à différentes questions conformément à leur expérience professionnelle.

Alain Devers et Michel Farge étudient les conséquences en droit international privé des phénomènes de déjudiciarisation (déjudiciarisation de la séparation de corps par consentement mutuel et déjudiciarisation de la révision de la contribution à l'éducation et à l'entretien des enfants, déjudiciarisation du changement de régime matrimonial) et de simplification du droit interne de la famille. Une partie de cette contribution prend en considération les évolutions apportées au droit international privé du divorce par le règlement $n^{\circ}$ 2019/1111 Bruxelles II ter.

Edihno Dos-Reis aborde la question de l'enfant dans la perspective du divorce de ses parents. L'Auteur souligne que le législateur contemporain préserve des relations parent—enfant: le couple parental, même s'il peut divorcer librement dans les conditions prévues par la loi, doit continuer « à assumer de concert la charge éducative de l'enfant» [p. 154]. Ainsi, d'une part, cette préservation se fait par le truchement des règles procédurales permettant à écarter les enfants de la procédure de divorce de leurs parents (la réservation de l'action en divorce aux seuls parents, l'interdiction du témoignage des descendants). Elle s'instaure d'autre part, par le biais de règles substantielles en faveur d'une coparentalité réelle (la possibilité pour le juge de fixer la résidence de l'enfant en alternance, le droit de visite et d'hébergement au cas d'un exercice unilatéral de l'autorité parentale et autres).

Sylvain Bernard se concentre sur le problème de la prestation compensatoire « oubliée de la loi de modernisation de justice» [p. 165]. Effectivement, la loi $\mathrm{n}^{\mathrm{o}} 2016-1547 \mathrm{du} 18$ novembre 2016 ne donne aucune disposition ni réforme quant à l'existence et l'évaluation de cette indemnité compensatrice qui ne semble pas adaptée aux mutations contemporaines de la famille. L'Auteur énonce que 
plusieurs facteurs mènent à craindre la défaillance de la compensation des disparités économiques: d'un côté, il s'agit des fondements de cette indemnité, d'autre côté, des difficultés liées à son évaluation par des formules mathématiques.

Pierre Murat, dans ses propos résumant la thématique de l'ouvrage, rappelle deux notions fondamentales: la libéralisation et la déjudiciarisation résultant de l'augmentation du rôle reconnu aux volontés individuelles. Effectivement, pendant longtemps le législateur favorisait un modèle juridique: le mariage. Actuellement, le droit prend en compte une multiplication des situations résultant des volontés individuelles: l'existence ou non d'un couple, la structure du couple (personnes mariées, partenaires ou vivant en concubinage), la nature sexuelle du couple, les réalités d'engendrement (au sein du couple, au moyen de la PMA et de la GPA), le rôle de l'adulte dans l'éducation (surtout en ce qui concerne le parent d'intention, l'adoption plénière au sein d'un couple non marié), le sexe de celui qui conçoit (les liens entre le changement de sexe et la filiation juridique). À part cela, l'Auteur énumère trois sources pour circonscrire le concept de filiation: la filiation biologique, l'adoption et « la volonté d'avoir fait naître un enfant alors que celui-ci n'a pas de lien biologique avec l'auteur de cette volonté» [p. 195]. L'intérêt de l'enfant n'y est pas à négliger. En ce qui concerne la question de la déjudiciarisation, l'Auteur souligne le risque d'une simplification trop poussée de la profession d'avocat « qui pourrait ne pas mener à une amélioration de la justice» [p. 204]. De plus, on observe une promotion du domaine contractuel dans le droit de la famille et les difficultés qui risquent d'arriver si la convention est annulée.

Pour conclure, cet ouvrage présente un éventail des changements touchant le droit de la famille en France sous un angle pluridisciplinaire, dans la perspective pratique et théorique. Il peut constituer une référence pour les spécialistes en droit, les étudiants et les enseignants intéressés par la problématique abordée toujours actuelle et évolutive.

Table des matières.

Partie 1. La fonction symbolique du droit de la famille.

Titre 1. La question du sexe, p. 9.

Loïc Bret, Sexe et genre. Réflexions sur la place de la volonté individuelle dans l'état civil, p. 11.

Titre 2. La question de la filiation, p. 19.

Jennifer Naselli, AMP / GPA: l'état du droit positif après vingt-cinq ans de gestation, p. 23.

Jehanne Sosson et Hélène Malmanche, État du droit belge en matière de procréation médicalement assistée et de gestation pour autrui, p. 37.

Géraldine Vial, Le réexamen des décisions civiles rendues en matière de gestation pour autrui. Procédure, enjeux et perspectives, p. 61. 
Partie 2. La fonction opératoire du droit de la famille.

Titre 1. La déjudiciarisation du divorce, p. 71.

Nicolas Frémeaux, Mariage et patrimoine. Quelles évolutions récentes et quelles conséquences ?, p. 73.

Ingrid Maria, Divorce sans juge. État des lieux du droit positif., p. 87.

Mikaël Gros, La remise en cause du divorce déjudiciarisé par le droit commun des contrats, p. 97.

Ingrid Maria, Les pouvoirs du JEX sur le titre contractuel. Étude de contentieux, p. 111.

Frédéric Hébert et Jean-Luc Charras, Premier bilan sur la pratique du divorce conventionnel, le regard du notaire, p. 125.

Alain Devers et Michel Farge, La déjudiciarisation et la simplification du droit de la famille. Conséquences en droit international privé, p.135.

Titre 2. Les conséquences du divorce: vers d'autres réformes ?, p. 151.

Edihno Dos-Reis, L'enfant et le divorce de ses parents, p. 153.

Sylvain Bernard, Disparité économique et rupture du couple. Vers une réforme de la prestation compensatoire?, p. 165.

Pierre Murat, Propos conclusifs. Soubresauts législatifs ou changement de paradigme pour la famille et son droit ?, p. 185.

Index, p. 207.

Open Access This article is licensed under a Creative Commons Attribution 4.0 International License, which permits use, sharing, adaptation, distribution and reproduction in any medium or format, as long as you give appropriate credit to the original author(s) and the source, provide a link to the Creative Commons licence, and indicate if changes were made. The images or other third party material in this article are included in the article's Creative Commons licence, unless indicated otherwise in a credit line to the material. If material is not included in the article's Creative Commons licence and your intended use is not permitted by statutory regulation or exceeds the permitted use, you will need to obtain permission directly from the copyright holder. To view a copy of this licence, visit http://creativecommons.org/licen ses/by/4.0/.

Publisher's Note Springer Nature remains neutral with regard to jurisdictional claims in published maps and institutional affiliations. 